\title{
THE HOSPITAL BURDEN OF INTERGENERATIONAL CONTACT WITH THE WELFARE SYSTEM: \\ A whole-of-population liked data study
}

Alexandra M Procter, PhD Candidate ${ }^{1,2}$, Catherine R Chittleborough, Senior Lecturer ${ }^{1,2}$, Rhiannon M Pilkington, Postdoctoral Researcher ${ }^{1,2}$, Odette Pearson, Senior Research Fellow $^{3,4}$, Alicia Montgomerie, Research Associate ${ }^{1,2}$, John W Lynch, Professor ${ }^{1,2,5}$

${ }^{1}$ School of Public Health, The University of Adelaide, Adelaide, 5005, South Australia

${ }^{2}$ Robinson Research Institute, The University of Adelaide, Adelaide, 5005, South Australia

${ }^{3}$ Wardliparingga Aboriginal Health Research Unit, South Australian Health and Medical Research Institute, Adelaide, 5000, South Australia

${ }^{4}$ Adelaide Medical School, The University of Adelaide, Adelaide, 5005, South Australia

${ }^{5}$ Population Health Sciences, University of Bristol, Bristol, BS8 1QU, United Kingdom

\section{CORRESPONDENCE TO:}

Alexandra Marie Procter

School of Public Health, The University of Adelaide

Rundle Mall Plaza, Level 5

50 Rundle Mall, Adelaide

South Australia 5005, Australia

Telephone: +61-8-8313-3851

Email: alexandra.procter@adelaide.edu.au

WORD COUNT: 3,553 


\section{AUTHOR'S CONTRIBUTIONS}

Conceptualisation, AMP, CRC, RMP, OP and JWL; Methodology, AMP, CRC, RMP, AM, and JWL; Data curation- AMP, RMP, and AM Formal Analysis, AMP; data interpretation, AMP, CRC, RMP, OP and JWL; Writing - Original Draft, AMP; Writing - Review and Editing, AMP, CRC, RMP, OP, AM, and JWL; Supervision, CRC, RMP, and JWL; Funding Acquisition, JWL and AMP. The corresponding author (AMP) attests that all listed authors meet authorship criteria and that no others meeting the criteria have been omitted.

\section{CONFLICT OF INTEREST DECLARATION}

The Authors declare no conflicts of interest.

\section{ROLE OF FUNDING SOURCE}

JWL was awarded a National Health and Medical Research Council (NHMRC) Australia Fellowship (570120); and NHMRC Centre of Research Excellence (1099422); JWL, CRC was awarded an NHMRC Partnership Project Grant (1056888); RMP, AM, was supported by funds from the NHMRC Centre for Research Excellence; OP was awarded a Medical Research Future Fund (MRFF) Primary Health Care Research Initiative Grant (12300314) and an NHMRC Ideas Grant (013029); and AMP was supported by funds from the Westpac Scholars Trust.

The funding sources had no role in the analysis or writing of the manuscript nor the decision to submit for publication.

\section{ETHICS COMMITTEE APPROVAL}

Ethics approval for use of this data was granted from the University of Adelaide (H-1852011), the SA Department of Health and Wellbeing (HREC/13/SAH/106), and the AIHW Ethics Committee (EO2017/4/396). Site-Specific Approval for use of hospital data was given 
medRxiv preprint doi: https://doi.org/10.1101/2021.11.15.21266370; this version posted November 16, 2021. The copyright holder for this preprint (which was not certified by peer review) is the author/funder, who has granted medRxiv a license to display the preprint in perpetuity. It is made available under a CC-BY-NC-ND 4.0 International license .

by SA Department of Health and Wellbeing (Central SSA/13/SAH/146) and Women's and Children's Health Network (SSA/14/WCHN/21). 


\begin{abstract}
Background: Intergenerational welfare contact (IWC) is a policy issue because of the personal and social costs of intergenerational disadvantage. We estimated the hospital burden of IWC for children aged 11-20 years.
\end{abstract}

Methods: This linked data study of children born in South Australia, 1991-1995 ( $\mathrm{n}=94,358)$, and their parent/s (n=143,814) used de-identified data from the Better Evidence Better Outcomes Linked Data platform. Using Australian Government Centrelink data, welfare contact (WC) was defined as parent/s receiving a means-tested welfare payment (lowincome, unemployment, disability or caring) when children were aged 11-15, or children receiving payment at ages 16-20. IWC was WC occurring in both parent and child generations. Children were classified as: No WC, parent only WC, child only WC, or IWC. Hospitalisation rates and cumulative incidence were estimated by age and WC group.

Findings: IWC affected $34.9 \%$ of children, who had the highest hospitalisation rate (133.5 per 1,000 person-years) compared to no WC (46.1 per 1,000 person-years), parent only WC (75.0 per 1,000 person-years), and child only WC (87.6 per 1,000 person-years). Of all IWC children, $43.0 \%$ experienced at least one hospitalisation between 11-20, frequently related to injury, mental health, and pregnancy.

Interpretation: Children experiencing IWC represent a third of the population aged 11-20. Compared to children with parent-only WC, IWC children had 78\% higher hospitalisation rates from age 11 to 20 , accounting for over half of all hospitalisations in this age group. Frequent IWC hospitalisation causes were injuries, mental health, and pregnancy.

Funding: Medical Research Future Fund, National Health and Medical Research Council, Westpac Scholars Trust. 


\section{INTRODUCTION}

In 1848, Engels ${ }^{1}$ questioned "How is it possible, under such conditions, for the lower class to be healthy and long lived?". While there have been major population health advances since the $19^{\text {th }}$ century, health gaps between rich and poor remain stubbornly entrenched even in modern welfare states. ${ }^{2}$ In 2017-18, 1 in 6 Australian children under the age of 15 were living in poverty - less than half the population median household income. ${ }^{3}$ Growing up in socioeconomic disadvantage negatively affects child development, education, labour market, and health outcomes, over a life course and across generations. ${ }^{4-6}$ Income provides access to resources such as nutrition, housing, and medical care which directly and indirectly affect well-being, and thus income is a major social determinant of health. ${ }^{4,7}$ In high and middleincome countries welfare systems have the potential to buffer the effects of low income and poverty. Welfare systems, including Centrelink in Australia, aim to transfer income to support those experiencing low income due to joblessness, disability, retirement, and caring responsibilities. ${ }^{9,10}$ Payments are intended to help overcome the income loss from these circumstances, and in some cases foster financial independence through income support for further education. ${ }^{9,11}$ Over $40 \%$ of Australia's cash transfers are targeted at the lowest income quintile, making it the second highest targeted cash transfer system of all OECD countries.

Such a highly targeted welfare system supports the notion that welfare receipt is closely related to ongoing poverty and social exclusion. ${ }^{12}$ There is recent policy concern ${ }^{13}$ regarding families who become "trapped" in a cycle of intergenerational welfare contact (IWC). For government and non-government organisations, the policy discussion has been about breaking these cycles of disadvantage. ${ }^{13,14}$

The idea that reliance on welfare is transmitted from one generation to the next is not unique to Australia ${ }^{11,15,16}$ and has been documented in the United States, ${ }^{17,18}$ Canada, ${ }^{17}$ Nordic countries, ${ }^{19,20}$ and United Kingdom. ${ }^{21}$ Despite considerable research documenting that low 
income is associated with poor health, ${ }^{4,7,8,22}$ there are few studies that examine IWC and health. ${ }^{11,23}$ One study investigated the mediating role of health (limited to BMI, self-reported mental health and physical exercise) in IWC but did not report health outcomes for IWC children. ${ }^{23}$ Previous studies reporting that welfare contact (WC) is associated with poorer health are limited by quality (self-reported measures, ${ }^{24,25}$ selective sample populations, ${ }^{23-26}$ no longitudinal design ${ }^{15}$ ), lack of intergenerational perspective, ${ }^{24,26-30}$ and WC definitions that include any payment type such as those considered near universal. ${ }^{11,25-30}$ The policy problem of IWC in Australia has been examined by labour economist Cobb-Clark and colleagues (2017) who defined and measured IWC to better understand how disadvantage is transmitted from parents to children. ${ }^{15}$ Using welfare payment eligibility criteria we build on this to develop our preferred definition of means-tested WC that includes welfare payments which are intended to support individuals maintaining a basic standard of living.

The objective of our study is descriptive. ${ }^{31}$ We use whole-of-population linked administrative data that contains Australian government welfare payments linked to jurisdictional inpatient hospitalisations. To our knowledge, this is the first time federal government welfare payment data has been linked to state hospital data in Australia. This linked data study examined the proportion of almost 100,000 South Australian (SA) children born 1991-1995 who experienced IWC and other WC types; and their cause-specific hospitalisation experience from ages 11-20 years. We measured WC for parents when their children were aged 11-15 years, and for the children themselves receiving payment at ages 16-20 years. These birth cohorts and age ranges were selected to maximise the exposure (WC) and outcome (hospitalisation) windows given the data structure involving multiple birth cohorts (Figure 1). While the data platform used is whole-of-population, we acknowledge that this research has not included governance by Aboriginal and Torres Strait Islander people and therefore we chose not to include data of Aboriginal children and families. ${ }^{32}$ 


\section{METHODS}

\section{Data sources}

The data used for this study were part of the BEBOLD (Better Evidence Better Outcomes through Linked Data) platform which is a whole-of-population data resource including health, education, housing, and welfare systems administrative data. ${ }^{33}$ We used inpatient hospitalisations (Integrated SA Activity Collection), SA Perinatal Statistics Collection, the SA Births, Death and Marriages Registry, the family file (linking parents to children through birth registrations), and Centrelink data. Probabilistic linkage was performed by SA-NT DataLink, an independent third-party linkage agency. ${ }^{34}$ Australian data linkage systems estimate false linkage rates from $0.1 \%{ }^{35}$ to $0.5 \%{ }^{36}$ Centrelink data (Data Over Multiple INdividual Occurrences, DOMINO ${ }^{37}$ ) were linked as part of the BEBOLD Project by the Australian Institute of Health and Welfare (AIHW). DOMINO contains de-identified individual event-based data to provide a longitudinal picture of individual welfare payments by the Commonwealth Department of Social Services. ${ }^{37}$

\section{Ethics}

Ethics approval for use of this data was granted from the University of Adelaide (H-1852011), the SA Department of Health and Wellbeing (HREC/13/SAH/106), and the AIHW Ethics Committee (EO2017/4/396). Site-Specific Approval for use of hospital data was given by SA Department of Health and Wellbeing (Central SSA/13/SAH/146) and Women's and Children's Health Network (SSA/14/WCHN/21).

\section{Population}

Children in SA born 1991-1995 (n=98,571) were eligible to be included in the study (Figure 2). Individuals were identified as Aboriginal and/or Torres Strait Islander if they had at least one record as Aboriginal and/or Torres Strait Islander across inpatient hospitalisations, SA 
Perinatal Statistics, Centrelink demographics (DOMINO) and SA Births Registry. ${ }^{38}$ We made a conscious decision, in consultation with Aboriginal and Torres Strait Islander co-author and informed by the principles of Indigenous Data Sovereignty, not include Aboriginal and Torres Strait Islander children $(n=4,103,4.2 \%) \cdot{ }^{32}$ A further 110 children were excluded as they did not have a birth registration with at least one parent recorded, and $n \leq 5$ parents were excluded as they were unable to be linked due to missing information. Analysis was undertaken on children who were neither Aboriginal nor Torres Strait Islander ( $\mathrm{n}=94,358)$ and their parents $(n=143,814)$. We defined children's parent/s using birth registration data, where mother and (if acknowledged) co-parent was recorded. Of the children included in the analysis, $\mathrm{n}=91,192(96.6 \%)$ had both a mother and co-parent recorded in birth registration data and $n=3,166(3.4 \%)$ had one parent recorded.

\section{Hospitalisations}

Inpatient hospitalisation data records age at admission and the International Statistical Classification of Diseases and Related Health Problems, Tenth Revision, Australian Modification (ICD-10-AM) code/s for the admission. ${ }^{39}$ Hospitalisation type by chapter heading of the ICD-10-AM was defined using the primary diagnosis ICD-10-AM code recorded for the admission. ${ }^{39}$ Potentially preventable hospitalisations (PPHs) were calculated under a child-centric definition for admission at ages 11-14 and under the standard Australian definition (best suited to adults) at ages $15-20 .{ }^{40}$ Primary diagnosis codes used to identify paediatric Complex Chronic Conditions (CCCs) were adapted to the ICD-10-AM coding system. $^{41}$

\section{Welfare contact}

WC was defined as having received a Centrelink payment where the payment amount was greater than $\$ 0$ and the payment type (e.g. Youth Allowance, Newstart etc.) was recorded. 
Only $0.003 \%$ of payments had a missing payment type. Parent WC was defined as parents having WC when children were aged 11-15 years, and child WC as the child themselves having WC when aged 16-20 years (Figure 1). IWC was WC occurring in both parent and child generations. Children were categorised in one of four WC groups: No WC, parent only WC, child only WC and IWC.

We examined WC under two constructs: any WC, and 'means-tested' WC that only included payments subject to an income and assets test and aimed to assist those experiencing low income or hardship. Any WC included all payment types including those payments which may be considered 'near universal' for families with children (e.g. Family Tax Benefit, Baby Bonus, and Child Care Benefit). Our definition of means-tested WC included: Carer Allowance, Carer Payment, Disability Support Pension (DSP), Parenting payment partnered, Parenting payment single, Newstart Allowance, Newstart Mature Age Allowance, Youth Allowance, Partner allowance, and Wife Pension DSP (wife of a disability support pension). The intent of these payment types is detailed in Supplementary Table 1.

\section{Analysis}

For each birth cohort, we calculated the proportion of children who experienced no WC, parent only WC, child only WC and IWC, under both welfare constructs (any WC and means-tested WC).

All cause age-specific and overall admission rates for children aged 11-25 years were calculated by dividing the number of admissions by person-years. Cumulative incidence was the proportion of children who had experienced a hospital admission from age 11 years up to age 20 years. Hospitalisation admission rates and proportion of children experiencing hospitalisations by ICD-10-AM chapter, PPHs, and CCCs were calculated for ages 11-15 years, when children could have been exposed to parent WC, and 16-20 years, reflecting the 
age group when children could have experienced their own WC. Hospitalisation outcomes were reported across WC types under the means-tested welfare construct.

\section{Sensitivity analyses}

Given the data structure and the years of observation available, we undertook sensitivity analysis to assess potential misclassification due to the limited observation windows for both parent and child WC under the means-tested welfare construct. For children born in 1991, we examined child WC for an extended period (ages 16-24 years) and compared this to child WC at 16-20 years as used in the main analyses. For children born in 1995 we examined parent WC for an extended period (ages 7-15 years) and compared this to parent WC at 11-15 years as in the main analyses. For children born in both 1991 and 1995 we recreated the four WC groups using the extended period of parent or child WC.

Given family structures can change over time, we calculated the proportion of children whose parent/s were defined by birth registration (recorded at the time of birth) compared to parent/s recorded in the Centrelink relationships file (recorded at time of payment receipt). We defined 'parents' in the Centrelink relationships file as parent, grandparent or guardian relationships associated with the child. Gender of Centrelink parent relationship were defined using the gender recorded in the Centrelink demographics file. This sensitivity analysis was undertaken on two birth cohorts: children born 1995 and children born 2002.

Analyses were undertaken using STATA MP Version 16, 64-bit (StataCorp, College Station, TX, USA). 


\section{RESULTS}

\section{Welfare contact}

Table 1 shows that using any type of welfare payment nearly half $(48.9 \%, n=46,147)$ of children in SA born 1991-1995 experienced IWC, and only $9.8 \%(n=9,269)$ experienced no WC. Using the means-tested definition, IWC was experienced by a third of children $(34.9 \%$, $\mathrm{n}=32,969$ ), with $39.7 \%$ of children experiencing no $\mathrm{WC}, 10.9 \%$ parent only $\mathrm{WC}$, and $14.4 \%$ child only WC.

\begin{tabular}{|c|c|c|c|c|c|c|c|c|c|}
\hline \multirow[t]{2}{*}{$\begin{array}{l}\text { Welfare construct } \\
\text { Year of birth }\end{array}$} & \multicolumn{2}{|c|}{ No WC } & \multicolumn{2}{|c|}{ Parent only WC } & \multicolumn{2}{|c|}{ Child only WC } & \multicolumn{2}{|c|}{$\begin{array}{c}\text { Intergenerational } \\
\text { WC }\end{array}$} & \multirow{2}{*}{$\begin{array}{c}\text { Total } \\
\mathrm{n} \\
\end{array}$} \\
\hline & $\mathrm{n}$ & $\%$ & $\mathrm{n}$ & $\%$ & $\mathrm{n}$ & $\%$ & $\mathrm{n}$ & $\%$ & \\
\hline Any payment & & & & & & & & & \\
\hline 1991 & 1,988 & 10.6 & 7,438 & 39.7 & 437 & 2.3 & 8,881 & 47.4 & 18,744 \\
\hline 1992 & 1,911 & 10.0 & 7,536 & 39.4 & 339 & 1.8 & 9,335 & 48.8 & 19,121 \\
\hline 1993 & 1,772 & 9.3 & 7,558 & 39.8 & 294 & 1.5 & 9,388 & 49.4 & 19,012 \\
\hline 1994 & 1,815 & 9.6 & 7,226 & 38.4 & 268 & 1.4 & 9,525 & 50.6 & 18,834 \\
\hline 1995 & 1,783 & 9.6 & 7,584 & 40.7 & 262 & 1.4 & 9,018 & 48.4 & 18,647 \\
\hline 1991-1995 & 9,269 & 9.8 & 37,342 & 39.6 & 1,600 & 1.7 & 46,147 & 48.9 & 94,358 \\
\hline Means-tested* & & & & & & & & & \\
\hline 1991 & 7,470 & 39.9 & 2,231 & 11.9 & 2,517 & 13.4 & 6,526 & 34.8 & 18,744 \\
\hline 1992 & 7,554 & 39.5 & 2,193 & 11.5 & 2,673 & 14.0 & 6,701 & 35.0 & 19,121 \\
\hline 1993 & 7,477 & 39.3 & 2,078 & 10.9 & 2,858 & 15.0 & 6,599 & 34.7 & 19,012 \\
\hline 1994 & 7,397 & 39.3 & 1,845 & 9.8 & 2,835 & 15.1 & 6,757 & 35.9 & 18,834 \\
\hline 1995 & 7,609 & 40.8 & 1,913 & 10.3 & 2,739 & 14.7 & 6,386 & 34.2 & 18,647 \\
\hline 1991-1995 & 37,507 & 39.7 & 10,260 & 10.9 & 13,622 & 14.4 & 32,969 & 34.9 & 94,358 \\
\hline
\end{tabular}

Table 1: Welfare contact (WC) for children born 1991-1995, $\mathrm{n}$ individuals $=94,358$

\footnotetext{
*Means-tested contains the following payment types: Carer Allowance, Carer Payment, Disability Support Pension, Newstart Allowance, Newstart Mature Age Allowance, Parenting payment partnered, Parenting payment single, Partner allowance. Wife Pension Disability Support Pension (wife of a Disability Support pension), Youth Allowance.
}

\section{All cause hospitalisations}

From the perspective of health system burden as 'incidents' of hospitalisation, Table 2 shows the all-cause hospitalisation rate among 11-25 year olds was 46.1 per 1,000 person-years among those with no WC and 133.5 per 1,000 person-years for children with IWC. For every 
medRxiv preprint doi: https://doi.org/10.1101/2021.11.15.21266370; this version posted November 16, 2021. The copyright holder for this preprint (which was not certified by peer review) is the author/funder, who has granted medRxiv a license to display the preprint in perpetuity.

It is made available under a CC-BY-NC-ND 4.0 International license .

age, IWC children had higher admission rates compared to children experiencing no WC, or

WC in only one generation, with the exception of age 11 when IWC and parent only WC had similar hospitalisation rates. 
Table 2: All cause hospital admission rates per 1,000 person-years (py) for children born 1991-1995 by welfare contact (WC), $n$ individuals $=94,358 ; \mathrm{n}$ admissions $=105,250$

\begin{tabular}{|c|c|c|c|c|c|c|c|c|c|c|c|c|c|c|c|c|}
\hline \multirow{3}{*}{$\begin{array}{c}\text { Birth } \\
\text { cohorts }\end{array}$} & \multirow{3}{*}{$\begin{array}{l}\text { Age } \\
\text { years }\end{array}$} & \multicolumn{3}{|c|}{ No WC } & \multicolumn{3}{|c|}{ Parent only WC } & \multicolumn{3}{|c|}{ Child only WC } & \multicolumn{3}{|c|}{ Intergenerational WC } & \multicolumn{3}{|c|}{ Overall } \\
\hline & & & & $\begin{array}{c}\text { rate } \\
\text { per } \\
\mathbf{1 , 0 0 0}\end{array}$ & & & $\begin{array}{c}\text { rate } \\
\text { per } \\
\mathbf{1 , 0 0 0}\end{array}$ & & & $\begin{array}{c}\text { rate } \\
\text { per } \\
\mathbf{1 , 0 0 0}\end{array}$ & & & $\begin{array}{c}\text { rate } \\
\text { per } \\
1,000\end{array}$ & & & $\begin{array}{c}\text { rate } \\
\text { per } \\
\mathbf{1 , 0 0 0}\end{array}$ \\
\hline & & py & $\mathrm{n}$ & py & py & $\mathrm{n}$ & py & py & $\mathrm{n}$ & py & py & $\mathrm{n}$ & py & py & $\mathrm{n}$ & py \\
\hline 1991-1995 & 11 & 37,507 & 1,066 & 28.4 & 10,260 & 692 & 67.4 & 13,622 & 510 & 37.4 & 32,969 & 2,161 & 65.5 & 94,358 & 4,429 & 46.9 \\
\hline 1991-1995 & 12 & 37,507 & 1,187 & 31.6 & 10,260 & 615 & 59.9 & 13,622 & 613 & 45.0 & 32,969 & 2,184 & 66.2 & 94,358 & 4,599 & 48.7 \\
\hline 1991-1995 & 13 & 37,507 & 1,275 & 34.0 & 10,260 & 664 & 64.7 & 13,622 & 624 & 45.8 & 32,969 & 2,335 & 70.8 & 94,358 & 4,898 & 51.9 \\
\hline 1991-1995 & 14 & 37,507 & 1,377 & 36.7 & 10,260 & 766 & 74.7 & 13,622 & 687 & 50.4 & 32,969 & 2,845 & 86.3 & 94,358 & 5,675 & 60.1 \\
\hline 1991-1995 & 15 & 37,507 & 1,496 & 39.9 & 10,260 & 766 & 74.7 & 13,622 & 855 & 62.8 & 32,969 & 3,401 & 103.2 & 94,358 & 6,518 & 69.1 \\
\hline 1991-1995 & 16 & 37,507 & 1,669 & 44.5 & 10,260 & 732 & 71.3 & 13,622 & 1,275 & 93.6 & 32,969 & 4,275 & 129.7 & 94,358 & 7,951 & 84.3 \\
\hline 1991-1995 & 17 & 37,507 & 1,892 & 50.4 & 10,260 & 874 & 85.2 & 13,622 & 1,339 & 98.3 & 32,969 & 4,842 & 146.9 & 94,358 & 8,947 & 94.8 \\
\hline 1991-1995 & 18 & 37,507 & 1,969 & 52.5 & 10,260 & 707 & 68.9 & 13,622 & 1,463 & 107.4 & 32,969 & 5,242 & 159.0 & 94,358 & 9,381 & 99.4 \\
\hline 1991-1995 & 19 & 37,507 & 1,954 & 52.1 & 10,260 & 724 & 70.6 & 13,622 & 1,546 & 113.5 & 32,969 & 5,637 & 171.0 & 94,358 & 9,861 & 104.5 \\
\hline 1991-1995 & 20 & 37,507 & 1,974 & 52.6 & 10,260 & 771 & 75.1 & 13,622 & 1,667 & 122.4 & 32,969 & 5,691 & 172.6 & 94,358 & 10,103 & 107.1 \\
\hline 1991-1995 & 21 & 37,507 & 2,130 & 56.8 & 10,260 & 851 & 82.9 & 13,622 & 1,747 & 128.2 & 32,969 & 5,568 & 168.9 & 94,358 & 10,296 & 109.1 \\
\hline 1991-1994 & 22 & 29,898 & 1,650 & 55.2 & 8,347 & 730 & 87.5 & 10,883 & 1,268 & 116.5 & 26,583 & 4,917 & 185.0 & 75,711 & 8,565 & 113.1 \\
\hline 1991-1993 & 23 & 22,501 & 1,372 & 61.0 & 6,502 & 643 & 98.9 & 8,048 & 952 & 118.3 & 19,826 & 3,903 & 196.9 & 56,877 & 6,870 & 120.8 \\
\hline 1991-1992 & 24 & 15,024 & 916 & 61.0 & 4,424 & 360 & 81.4 & 5,190 & 621 & 119.7 & 13,227 & 2,774 & 209.7 & 37,865 & 4,671 & 123.4 \\
\hline 1991 & 25 & 7,470 & 523 & 70.0 & 2,231 & 189 & 84.7 & 2,517 & 286 & 113.6 & 6,526 & 1,488 & 228.0 & 18,744 & 2,486 & 132.6 \\
\hline Total & $11-25$ & 487,470 & 22,450 & 46.1 & 134,364 & 10,084 & 75.0 & 176,480 & 15,453 & 87.6 & 428,821 & 57,263 & 133.5 & $1,227,135$ & 105,250 & 85.8 \\
\hline
\end{tabular}


medRxiv preprint doi: https://doi.org/10.1101/2021.11.15.21266370; this version posted November 16, 2021. The copyright holder for this preprint (which was not certified by peer review) is the author/funder, who has granted medRxiv a license to display the preprint in perpetuity.

It is made available under a CC-BY-NC-ND 4.0 International license .

From the perspective of a cohort of children experiencing hospitalisation, Table 3 shows that the cumulative 'incidence' (proportion of children hospitalised at least once) from ages 11 to 20 years was highest for IWC children (43.0\%) compared to children experiencing no WC (23.7\%), parent only WC (29.3\%), or child only WC (34.3\%).

Table 3: Cumulative incidence of all cause hospitalisation by welfare contact (WC) and age, $\mathrm{n}$ individuals $=94,358$

\begin{tabular}{|c|c|c|c|c|c|c|c|c|c|c|}
\hline \multirow[t]{2}{*}{$\begin{array}{c}\text { Age } \\
\text { years }\end{array}$} & \multicolumn{2}{|c|}{$\begin{array}{c}\text { No WC } \\
n \text { individuals }=37,507\end{array}$} & \multicolumn{2}{|c|}{$\begin{array}{c}\text { Parent only WC } \\
\mathrm{n} \text { individuals }=10,260\end{array}$} & \multicolumn{2}{|c|}{$\begin{array}{c}\text { Child only WC } \\
\mathrm{n} \text { individuals }=13,622\end{array}$} & \multicolumn{2}{|c|}{$\begin{array}{l}\text { Intergenerational WC } \\
\mathrm{n} \text { individuals }=32,969\end{array}$} & \multicolumn{2}{|c|}{$\begin{array}{c}\text { Overall } \\
\mathrm{n} \text { individuals }=94,358\end{array}$} \\
\hline & $\mathrm{n}$ & $\%$ & $\mathrm{n}$ & $\%$ & $\mathrm{n}$ & $\%$ & $\mathrm{n}$ & $\%$ & $\mathrm{n}$ & $\%$ \\
\hline 11 & 875 & 2.3 & 367 & 3.6 & 417 & 3.1 & 1,598 & 4.8 & 3,257 & 3.5 \\
\hline 12 & 1,734 & 4.6 & 671 & 6.5 & 847 & 6.2 & 2,853 & 8.7 & 6,105 & 6.5 \\
\hline 13 & 2,528 & 6.7 & 978 & 9.5 & 1,216 & 8.9 & 4,069 & 12.3 & 8,791 & 9.3 \\
\hline 14 & 3,404 & 9.1 & 1,263 & 12.3 & 1,610 & 11.8 & 5,404 & 16.4 & 11,681 & 12.4 \\
\hline 15 & 4,301 & 11.5 & 1,585 & 15.4 & 2,066 & 15.2 & 6,891 & 20.9 & 14,843 & 15.7 \\
\hline 16 & 5,219 & 13.9 & 1,898 & 18.5 & 2,577 & 18.9 & 8,461 & 25.7 & 18,155 & 19.2 \\
\hline 17 & 6,190 & 16.5 & 2,212 & 21.6 & 3,151 & 23.1 & 9,987 & 30.3 & 21,540 & 22.8 \\
\hline 18 & 7,128 & 19.0 & 2,506 & 24.4 & 3,728 & 27.4 & 11,526 & 35.0 & 24,888 & 26.4 \\
\hline 19 & 8,003 & 21.3 & 2,773 & 27.0 & 4,248 & 31.2 & 12,888 & 39.1 & 27,912 & 29.6 \\
\hline 20 & 8,873 & 23.7 & 3,009 & 29.3 & 4,679 & 34.3 & 14,190 & 43.0 & 30,751 & 32.6 \\
\hline
\end{tabular}

\section{Cause-specific hospitalisations}

The proportion of individuals with at least one hospitalisation by condition type (ICD-10-AM chapter heading, PPHs, and CCCs) and WC group is shown for hospitalisations at ages 11-15 years (Table 4) and 16-20 years (Table 5). The most common causes of admission were injuries, poisoning and external causes regardless of $\mathrm{WC}$ or age group. A higher proportion of IWC children experienced at least one hospitalisation related to respiratory conditions, mental health, digestive conditions, and pregnancy compared to any other type of WC for both age groups. For each of these causes the proportion was higher at ages 16-20 compared to 11-15 years (e.g. 'Mental and Behavioural disorders (FOO-F09)' age 11-15 years: 1.4\%; age 16-20 years: 3.7\%). IWC children had the highest proportion of PPHs compared to children experiencing all other WC types (age 11-15 years: 4.5\%; age 16-20 years: 4.8\%). Only a small proportion of IWC children experienced CCCs across both age groups (age 11-15 
medRxiv preprint doi: https://doi.org/10.1101/2021.11.15.21266370; this version posted November 16, 2021. The copyright holder for this preprint (which was not certified by peer review) is the author/funder, who has granted medRxiv a license to display the preprint in perpetuity.

It is made available under a CC-BY-NC-ND 4.0 International license .

years: $1.9 \%$; age $16-20$ years: $2.2 \%$ ). Similar patterns were observed when taking a health system perspective by reporting the cause-specific (ICD-10-AM chapter heading, PPHs and CCCs) admission rates per 1,000 person-years by WC and age group (Supplementary Tables 2 and 3). 
Table 4: Proportion of individuals with at least one hospitalisation by condition type and welfare contact (WC) for children born 19911995 , aged $11-15$ years, $\mathrm{n}$ individuals $=94,358$

\begin{tabular}{|c|c|c|c|c|c|c|c|c|c|c|}
\hline \multirow[t]{2}{*}{ Condition type (respective ICD-10-AM codes) } & \multicolumn{2}{|c|}{$\begin{array}{c}\text { No WC } \\
n \text { individuals }=37,507\end{array}$} & \multicolumn{2}{|c|}{$\begin{array}{c}\text { Parent only WC } \\
\mathrm{n} \text { individuals }=10,260\end{array}$} & \multicolumn{2}{|c|}{$\begin{array}{c}\text { Child only WC } \\
\mathrm{n} \text { individuals }=13,622\end{array}$} & \multicolumn{2}{|c|}{$\begin{array}{l}\text { Intergenerational WC } \\
\mathrm{n} \text { individuals }=32,969\end{array}$} & \multicolumn{2}{|c|}{$\begin{array}{c}\text { Overall } \\
\mathrm{n} \text { individuals }=94,358\end{array}$} \\
\hline & $\mathrm{n}$ & $\%$ & $\mathrm{n}$ & $\%$ & $\mathrm{n}$ & $\%$ & $\mathrm{n}$ & $\%$ & $\mathrm{n}$ & $\%$ \\
\hline Certain infectious \& parasitic diseases (A00-B99) & 283 & 0.8 & 109 & 1.1 & 128 & 0.9 & 425 & 1.3 & 945 & $\mathbf{1 . 0}$ \\
\hline Neoplasms (C00-D48) & 92 & 0.2 & 84 & 0.8 & 65 & 0.5 & 229 & 0.7 & 470 & 0.5 \\
\hline $\begin{array}{l}\text { Diseases of the blood and blood-forming organs } \\
\text { and certain disorders involving the immune }\end{array}$ & & & & & & & & & & \\
\hline $\begin{array}{l}\text { mechanism (D50-D89) } \\
\text { Endocrine, nutritional and metabolic disorders }\end{array}$ & 24 & 0.1 & 26 & 0.3 & 9 & 0.1 & 41 & 0.1 & 100 & 0.1 \\
\hline$(\mathrm{E} 00-\mathrm{E} 89)$ & 90 & 0.2 & 79 & 0.8 & 41 & 0.3 & 197 & 0.6 & 407 & 0.4 \\
\hline Mental and behavioural disorders (F00-F99) & 151 & 0.4 & 50 & 0.5 & 88 & 0.6 & 457 & 1.4 & 746 & 0.8 \\
\hline Diseases of the nervous system (G00-G99) & 112 & 0.3 & 51 & 0.5 & 52 & 0.4 & 285 & 0.9 & 500 & 0.5 \\
\hline Diseases of the eye and adnexa (H00-H59) & 34 & 0.1 & 8 & 0.1 & 17 & 0.1 & 87 & 0.3 & 146 & 0.2 \\
\hline Disease of the ear and mastoid process (H60-H95) & 71 & 0.2 & 41 & 0.4 & 48 & 0.4 & 216 & 0.7 & 376 & 0.4 \\
\hline Diseases of the circulatory system (I00-I99) & 71 & 0.2 & 34 & $\mathbf{0 . 3}$ & 34 & 0.2 & 120 & 0.4 & 259 & 0.3 \\
\hline Diseases of the respiratory system (J00-J99) & 504 & 1.3 & 222 & 2.2 & 318 & 2.3 & 1,171 & 3.6 & 2,215 & 2.3 \\
\hline Diseases of the digestive system (K00-K93) & 680 & 1.8 & 252 & 2.5 & 365 & 2.7 & 1,112 & 3.4 & 2,409 & 2.6 \\
\hline $\begin{array}{l}\text { Diseases of the skin and subcutaneous tissue } \\
\text { system (L00-L099) }\end{array}$ & 214 & 0.6 & 77 & 0.8 & 128 & 0.9 & 500 & 1.5 & 919 & 1.0 \\
\hline $\begin{array}{l}\text { Diseases of the musculoskeletal system and } \\
\text { connective tissue (M00-M99) }\end{array}$ & 205 & 0.5 & 73 & $\mathbf{0 . 7}$ & 106 & 0.8 & 385 & 1.2 & 769 & 0.8 \\
\hline Diseases of the genitourinary system (N00-N99) & 204 & 0.5 & 84 & 0.8 & 98 & $\mathbf{0 . 7}$ & 354 & 1.1 & 740 & 0.8 \\
\hline $\begin{array}{l}\text { Pregnancy, childbirth and the puerperium (O00- } \\
\text { O99) }\end{array}$ & 7 & $\mathbf{0 . 0}$ & 6 & 0.1 & 21 & 0.2 & 145 & 0.4 & 179 & 0.2 \\
\hline $\begin{array}{l}\text { Certain conditions originating in the perinatal } \\
\text { period (P00-P96) }\end{array}$ & 0 & $\mathbf{0 . 0}$ & 0 & $\mathbf{0 . 0}$ & 0 & $\mathbf{0 . 0}$ & 0 & $\mathbf{0 . 0}$ & 0 & $\mathbf{0 . 0}$ \\
\hline $\begin{array}{l}\text { Congenital malformations, deformations and } \\
\text { chromosomal abnormalities (Q00-Q99) }\end{array}$ & 87 & 0.2 & 37 & 0.4 & 39 & 0.3 & 205 & 0.6 & 368 & 0.4 \\
\hline Symptoms, signs and abnormal clinical and & & & & & & & & & & \\
\hline $\begin{array}{l}\text { laboratory findings, not elsewhere classified (R00- } \\
\text { R99) }\end{array}$ & 452 & 1.2 & 161 & 1.6 & 211 & 1.5 & 846 & 2.6 & 1,670 & 1.8 \\
\hline $\begin{array}{l}\text { Injury, poisoning and certain other consequences of } \\
\text { external causes (S00-T98) }\end{array}$ & 1,670 & 4.5 & 554 & 5.4 & 710 & 5.2 & 2,174 & 6.6 & 5,108 & 5.4 \\
\hline
\end{tabular}


Factors influencing health status and contact with health services (Z00-Z99)

Potentially preventable hospitalisations (PPHs)

$\frac{\text { Complex Chronic Conditions (CCCs) }}{\text { Note: children may experienc }}$

Note: children may experience multiple admissions and the

$\begin{array}{ll}208 & \text { 0. } \\ 726 & \mathbf{1} \\ 164 & \mathbf{0 .}\end{array}$

\begin{tabular}{l|l} 
& \\
.6 & \\
.4 &
\end{tabular}

\begin{tabular}{ll|} 
& \\
102 & $\mathbf{1 . 0}$ \\
296 & $\mathbf{2 . 9}$ \\
208 & $\mathbf{2 . 0}$
\end{tabular}

87
364
79

0.6
2.7

\begin{tabular}{l|r} 
& \\
$\mathbf{0 . 6}$ & 328 \\
$\mathbf{2 . 7}$ & 1,485 \\
$\mathbf{0 . 6}$ & 630 \\
\hline
\end{tabular}


Table 5: Proportion of individuals with at least one hospitalisation by condition type and welfare contact (WC) for children born 19911995, aged 16-20 years, $n$ individuals $=94,358$

\begin{tabular}{|c|c|c|c|c|c|c|c|c|c|c|}
\hline \multirow[t]{2}{*}{ Condition type (respective ICD-10-AM codes) } & \multicolumn{2}{|c|}{$\begin{array}{c}\text { No WC } \\
\mathrm{n} \text { individuals }=37,507\end{array}$} & \multicolumn{2}{|c|}{$\begin{array}{c}\text { Parent only WC } \\
\mathrm{n} \text { individuals }=10,260\end{array}$} & \multicolumn{2}{|c|}{$\begin{array}{c}\text { Child only WC } \\
\mathrm{n} \text { individuals }=13,622\end{array}$} & \multicolumn{2}{|c|}{$\begin{array}{c}\text { Intergenerational } \mathrm{WC} \\
\mathrm{n} \text { individuals }=32,969\end{array}$} & \multicolumn{2}{|c|}{$\begin{array}{c}\text { Overall } \\
\mathrm{n} \text { individuals }=94,358 \\
\end{array}$} \\
\hline & $\mathrm{n}$ & $\%$ & $\mathrm{n}$ & $\%$ & $\mathrm{n}$ & $\%$ & $\mathrm{n}$ & $\%$ & $\mathrm{n}$ & $\%$ \\
\hline Certain infectious \& parasitic diseases (A00-B99) & 402 & 1.1 & 115 & 1.1 & 208 & 1.5 & 570 & 1.7 & 1,295 & 1.4 \\
\hline Neoplasms (C00-D48) & 101 & 0.3 & 54 & 0.5 & 70 & 0.5 & 239 & 0.7 & 464 & 0.5 \\
\hline $\begin{array}{l}\text { Diseases of the blood and blood-forming organs } \\
\text { and certain disorders involving the immune }\end{array}$ & & & & & & & & & & \\
\hline mechanism (D50-D89) & 40 & 0.1 & 18 & 0.2 & 24 & 0.2 & 82 & 0.2 & 164 & 0.2 \\
\hline $\begin{array}{l}\text { Endocrine, nutritional and metabolic disorders } \\
\text { (E00-E89) }\end{array}$ & 99 & 0.3 & 68 & 0.7 & 48 & 0.4 & 211 & 0.6 & 426 & 0.5 \\
\hline Mental and behavioural disorders (F00-F99) & 466 & 1.2 & 145 & 1.4 & 371 & 2.7 & 1234 & 3.7 & 2,216 & 2.3 \\
\hline Diseases of the nervous system (G00-G99) & 110 & 0.3 & 47 & 0.5 & 80 & 0.6 & 309 & 0.9 & 546 & 0.6 \\
\hline Diseases of the eye and adnexa (H00-H59) & 30 & 0.1 & 11 & 0.1 & 19 & 0.1 & 72 & 0.2 & 132 & 0.1 \\
\hline Disease of the ear and mastoid process (H60-H95) & 30 & 0.1 & 16 & 0.2 & 28 & 0.2 & 94 & 0.3 & 168 & 0.2 \\
\hline Diseases of the circulatory system (I00-I99) & 101 & 0.3 & 45 & 0.4 & 66 & 0.5 & 185 & 0.6 & 397 & 0.4 \\
\hline Diseases of the respiratory system (J00-J99) & 641 & 1.7 & 256 & 2.5 & 410 & 3.0 & 1,257 & 3.8 & 2,564 & 2.7 \\
\hline Diseases of the digestive system (K00-K93) & 1185 & 3.2 & 369 & 3.6 & 796 & 5.8 & 2,075 & 6.3 & 4,425 & 4.7 \\
\hline $\begin{array}{l}\text { Diseases of the skin and subcutaneous tissue } \\
\text { system (L00-L099) }\end{array}$ & 315 & 0.8 & 138 & 1.3 & 168 & 1.2 & 673 & 2.0 & 1,294 & 1.4 \\
\hline $\begin{array}{l}\text { Diseases of the musculoskeletal system and } \\
\text { connective tissue (M00-M99) }\end{array}$ & 289 & 0.8 & 82 & 0.8 & 161 & 1.2 & 600 & 1.8 & 1132 & 1.2 \\
\hline Diseases of the genitourinary system (N00-N99) & 416 & 1.1 & 157 & 1.5 & 264 & 1.9 & 953 & 2.9 & 1790 & 1.9 \\
\hline $\begin{array}{l}\text { Pregnancy, childbirth and the puerperium (O00- } \\
\text { O99) }\end{array}$ & 308 & 0.8 & 153 & 1.5 & 608 & 4.5 & 2594 & 7.9 & 3663 & 3.9 \\
\hline $\begin{array}{l}\text { Certain conditions originating in the perinatal } \\
\text { period (P00-P96) }\end{array}$ & \# & \# & \# & \# & \# & \# & \# & \# & \# & \# \\
\hline $\begin{array}{l}\text { Congenital malformations, deformations and } \\
\text { chromosomal abnormalities (Q00-Q99) }\end{array}$ & \# & \# & \# & \# & \# & \# & \# & \# & \# & \# \\
\hline Symptoms, signs and abnormal clinical and & & & & & & & & & & \\
\hline $\begin{array}{l}\text { laboratory findings, not elsewhere classified (R00- } \\
\text { R99) }\end{array}$ & 639 & 1.7 & 254 & 2.5 & 446 & 3.3 & 1,547 & 4.7 & 2,886 & 3.1 \\
\hline $\begin{array}{l}\text { Injury, poisoning and certain other consequences of } \\
\text { external causes (S00-T98) }\end{array}$ & 1,887 & 5.0 & 634 & 6.2 & 994 & 7.3 & 2,961 & 9.0 & 6,476 & 6.9 \\
\hline
\end{tabular}


Factors influencing health status and contact with health services (Z00-Z99)

$\begin{array}{ll}255 & \mathbf{0 . 7} \\ 682 & \mathbf{1 . 8}\end{array}$

Potentially preventable hospitalisations (PPHs)

241

0.6

Chronic Conditions (CCCs)

Note: children may experience multiple admissions

\# represents cells concealed due to cell size $n<5$

200

713

2.2

1,308 


\section{Sensitivity analysis}

Both sensitivity analyses were consistent with the main findings.

Supplementary Table 4 compares child WC measured at 16-20 years to an extended view measured at 16-24 years for children born in 1991. Under the extended view, an additional $\mathrm{n}=2,204$ children ( $11.8 \%$ of the birth cohort) experienced child only WC and an additional $\mathrm{n}=677$ children $(3.6 \%$ of birth cohort) experienced IWC. Supplementary Table 5 compares parent WC measured for children born in 1995 when the child was 11-15 years to an extended view measured when the child was $7-15$ years. An additional $n=912$ children $(4.9 \%$ of the birth cohort) experienced parent only WC and an additional $\mathrm{n}=659$ children $(3.5 \%$ of the birth cohort) experienced IWC. Increasing observation periods of either parent or child WC increased the portion of the cohort who experiences IWC by $\sim 3.5 \%$.

Sensitivity analysis was undertaken for children born in 1991 and 1995 to compare parental family structure defined by birth registration data to male and female parents recorded in the Centrelink relationships file. For $96.9 \%$ of children who had a female parent recorded in the Centrelink relationships file, that female parent ID matched the mother on the birth registration. For $90 \%$ of children with at least one male parent registered in the Centrelink relationships file, that male parent ID matched the co-parent on the birth registration. Only $33 \%$ of co-parents in birth registrations were recorded in the Centrelink relationships file as having a relationship to the child.

\section{DISCUSSION}

This study showed that 1 in 3 children (34.9\%) born in SA from 1991-1995 experienced IWC defined as means-tested payments designed to support more disadvantaged families raising children, and those children themselves. This is perhaps unsurprising, given cross-sectional 
survey estimates suggest 1 in 6 Australian children under 15 live in poverty. ${ }^{3}$ Our findings provide a nuanced understanding of IWC as it considered welfare payments with eligibility requirements linked to socioeconomic disadvantage. If we took the same approach as previous research and considered any payment type, 1 in 2 children $(48.9 \%, n=46,147)$ had experienced IWC. However, this estimate includes near universal payments that normalise the experience of IWC in society. While it is important that such universal supports exist, they have little meaningful interpretation for considering potential interventions ${ }^{42}$ to help break cycles of intergenerational disadvantage which should focus on differences between children with parent only WC and IWC.

We believe this is the first study to report the health burden of IWC using actual welfare payment data. Children with IWC had a $78 \%$ higher hospitalisation rate compared to children with parent only WC. We compare IWC children to those experiencing parent only WC as they are the target group we would potentially intervene on to 'break the cycle' of IWC. ${ }^{42}$ Of all IWC children, 43\% experienced at least one hospitalisation between 11-20 years, most commonly related to injury, respiratory conditions, digestive conditions, mental health, and pregnancy. While welfare receipt has been associated with poorer mental health outcomes, ${ }^{11,24,26,29}$ our study showed IWC children experience even higher mental health hospitalisation rates compared to WC experienced only in one generation. We expected IWC children to have higher injury hospitalisation rates given injury hospitalisation is more frequent in children from the most disadvantaged backgrounds ${ }^{40,41}$ but this had not been quantified before. IWC children having higher hospitalisation rates related to pregnancy and childbirth reflects the inclusion of welfare payments associated with parenting. ${ }^{15}$ A higher proportion of IWC children experienced a PPH which are also associated with socioeconomic disadvantage. ${ }^{40}$ While IWC children were more likely to experience a hospitalisation relating to a $\mathrm{CCC}$, the overall proportion was small (age 11-15 years: $1.9 \%$; age $16-20$ years: $2.2 \%$ ). 
This suggests the majority of IWC children are not experiencing ongoing chronic conditions and are likely to be receiving welfare payments due to low income and other indicators of socioeconomic disadvantage rather than chronic poor health.

Children who had a parent with WC were 2.4 times as likely to experience their own WC compared to children with no parent WC. This finding is consistent with previous work both in Australia ${ }^{15}$ and internationally. ${ }^{18-20}$ Progress in breaking cycles of entrenched disadvantage is slow. ${ }^{13,43}$ We show a third of the population experiences IWC and that IWC children contribute a higher hospital burden compared to children who experience no, parent only or child only WC. This distinction is important. It suggests that breaking the intergenerational cycle of WC is important for reducing hospital burden.

\section{Aboriginal and Torres Strait Islander people and their data}

Aboriginal and Torres Strait Islander children and families are over-represented in the welfare system. ${ }^{9}$ Typically, the outcomes for Aboriginal and Torres Strait Islander children and families are compared to the outcomes of non-Indigenous children and families. This is done without any or very little consideration of the context of the phenomena of interest. The Australian welfare system was constructed to improve wellbeing but the conceptual model it was built on was not created for Aboriginal and Torres Strait Islander people. If we were to take an Aboriginal and Torres Strait Islander world view, we would need to use a different conceptual model of what 'welfare' means. Such a model would consider family and kinship structure, the sharing of resources, and understand that money does not necessarily have the same meaning, nor reflects the holistic concept of wellbeing, for Aboriginal and Torres Strait Islander families and communities. ${ }^{44}$ A collective decision was made after consultation among the author group, one of whom is an Aboriginal and Torres Strait Islander researcher, to apply the principles of Indigenous Data Sovereignty to control the Aboriginal and Torres 
Strait Islander data in the BEBOLD data platform. In this case, the South Australian Aboriginal community were not involved in informing this research and so their data were not included. Globally, Indigenous populations are enacting Indigenous Data Sovereignty which is, 'the rights of Indigenous Peoples to determine the means of collection, access, analysis, interpretation, management, dissemination and re-use of data pertaining to the Indigenous Peoples from whom it has been derived, or to whom it relates'. ${ }^{32}$ These principles are fundamental and should be considered and applied by all researchers.

\section{Limitations}

We defined WC as a parent or child having received an eligible payment. Consideration of the amount and duration of payment are topics for future research. However, while it may be possible that individuals only received payments for a short period of time this is unlikely given that payments included under the means-tested construct are intended for longer-term income support. Cobb-Clark and colleagues found the likelihood of children experiencing IWC was more closely related to whether the parent received a welfare payment at all, rather than the amount, type or intensity. ${ }^{15}$

Our study defined family structure at the time of childbirth using SA birth registrations, which contains a record of mother and co-parent (if acknowledged). Centrelink records contain additional information regarding relationship that can imply family structure (i.e. parent, grandparent, sibling etc.). We found a high proportion of mothers (96.9\%) and coparents $(90 \%)$ from birth registration matched the Centrelink relationships file where recorded. Only $33 \%$ of birth registration co-parents were recorded in the Centrelink relationships file. If we were to define family structure using the Centrelink relationships file it would not be possible to know which parent relationship reflected the primary caregiver, and our view of two parent families would be substantially limited. 
Our estimates of WC type and IWC were limited by the data available. Sensitivity analysis showed that by increasing observation periods of either parent or child WC, the proportion of the cohort who experienced IWC increased by $\sim 3.5 \%$. While there is no reason to suspect that the association between WC and hospitalisations would be different if we could examine the entire life course of the children, our estimates of IWC should be considered an underestimation.

Private hospital data are not available for linkage in SA but public hospitals account for $92 \%$ of admissions classified as an emergency and the only children's hospital in SA is a public hospital, ${ }^{45}$ suggesting that we have captured the majority of hospitalisations in our analysis. The exclusion of private hospital data may mean we under-estimated cumulative incidence, but we would not expect the associations between WC and hospital burden to differ between children attending public and private hospitals.

\section{CONCLUSIONS}

Our study examined the health burden of IWC using a definition of means-tested payments designed to support disadvantaged families and their children. Children with IWC had a 78\% higher hospitalisation rate compared to children with parent only WC, and 190\% higher than no WC. The hospital burden associated with IWC children is large, with $43 \%$ IWC children experiencing at least one hospitalisation between ages 11-20 years. Means-tested IWC children comprised one-third of the study population, but accounted for over half of all hospital admissions from ages 11-20. Common causes of hospitalisations included PPHs, injuries, mental health, pregnancy and childbirth. 


\section{REFERENCES}

1. Engels F. The Condition of the Working Class in England. tr. Henderson OW and Chaloner WH [1958]. Stanford California: Stanford University Press; 1848.

2. Mackenbach JP. Health inequalities: Persistence and change in European welfare states. New York: Oxford University Press; 2019.

3. Davidson P, Saunders P, Bradbury B, Wong M. Poverty in Australia 2020: Part 1 Overview. ACOSS/UNSW Poverty and Inequality Partnership Report No. 3. Sydney 2020.

4. Lynch J, Kaplan G. Socioeconomic Position. In: Berkman LF, Kawachi I, editors. Social Epidemiology. New York: Oxford University Press; 2000. p. 13-35.

5. Lai ETC, Wickham S, Law C, Whitehead M, Barr B, Taylor-Robinson D. Poverty dynamics and health in late childhood in the UK: evidence from the Millennium Cohort Study. Archives of Disease in Childhood. 2019:archdischild-2018-316702. DOI: 10.1136/archdischild-2018-316702.

6. Galobardes B, Lynch JW, Davey Smith G. Childhood Socioeconomic Circumstances and Cause-specific Mortality in Adulthood: Systematic Review and Interpretation. Epidemiologic Reviews. 2004;26(1):7-21.

7. Galobardes B, Shaw M, Lawlor DA, Lynch JW, Davey Smith G. Indicators of socioeconomic position (part 1). Journal of epidemiology and community health. 2006;60(1):7-12. DOI: 10.1136/jech.2004.023531.

8. World Health Organization. Commission on Social Determinants of Health. Closing the gap in a generation: health equity through action on the social determinants of health. Final Report of the Commission on Social Determinants of Health. Geneva: World Health Organization; 2008.

9. Australian Insitute of Health and Welfare. Australia's welfare series no. 13. AUS 214. Canberra: AIHW; 2017.

10. Department of Human Services. A guide to Australian Government payments 1 July19 September 2019; 2019.

11. Perales F, Higginson A, Baxter J, Western M, Zubrick SR, Mitrou F. Intergenerational Welfare Dependency in Australia: A Review of the Literature. Queensland: Life course centre, Institute for Social Science Research, The University of Queensland; 2014.

12. Whiteford P. The Australian Tax-Transfer System: Architecture and Outcomes. Economic Record. 2010;86(275):528-44. DOI: https://doi.org/10.1111/j.14754932.2010.00634.x.

13. Select Committee on Intergenerational Welfare Dependence. Inquiry into Intergenerational Welfare Dependence, Parliament of Australia. 2018 [cited 2018 September]. Available from: https://www.aph.gov.au/Parliamentary Business/Committees/House/Former Commit tees/Intergenerational_Welfare_Dependence/IGWD

14. Paul Ramsay Foundation. Helping Australians Defy Disadvantage. 2021. Available from: https://paulramsayfoundation.org.au/

15. Cobb-Clark DA, Dahmann S, Salamanca N, Zhu A. Intergenerational Disadvantage: Learning about Equal Opportunity from Social Assistance Receipt. Queensland: Life course centre, Institute for Social Science Research, The University of Queensland; 2017.

16. d'Addio AC. Intergenerational Transmission of Disadvantage: Mobility of immobility Across Generations? Paris: OECD Publishing; 2007. 
17. Corak M. Inequality from generation to generation: The United States in comparison. The economics of inequality, poverty, and discrimination in the 21 st century. 2013;1:107-26.

18. Page ME. New evidence on the intergenerational correlation in welfare participation. In: M Corak Editors, Generational income mobility in North America and Europe. Cambridge: Cambridge University Press; 2004.

19. Kauppinen TM, Angelin A, Lorentzen T, Bäckman O, Salonen T, Moisio P, et al. Social background and life-course risks as determinants of social assistance receipt among young adults in Sweden, Norway and Finland. Journal of European Social Policy. 2014;24(3):273-88.

20. Stenberg SA. Inheritance of welfare recipiency: an intergenerational study of social assistance recipiency in postwar Sweden. Journal of Marriage and Family. 2000;62(1):228-39.

21. Hills J. Good Times, Bad Times: The Welfare Myth of Them and Us (Revised Edition). Great Britain: Poliy Press, The University of Britian; 2017.

22. Galobardes B, Shaw M, Lawlor DA, Lynch JW, Davey Smith G. Indicators of socioeconomic position (part 2). Journal of epidemiology and community health. 2006;60(2):95-101. DOI: 10.1136/jech.2004.028092.

23. Bubonya M, Cobb-Clark D. Pathways of Disadvantage: Unpacking the Intergenerational Correlation in Welfare: Institute for Social Science Research, The University of Queensland; 2019.

24. Collie A, Sheehan L, Mcallister A. The Health of Disability Support Pension and Newstart Allowance Recipients. Melbourne: Monash University; 2019.

25. Cobb-Clark D, Sartbayeva A. The relationship between income-support history and the characteristics and outcomes of Australian youth: Outcomes of wave 2 of the Youth in Focus survey. Canberra, ACT: Australian National University.; 2010.

26. Spady DW, Schopflocher DP, Svenson LW, Thompson AH. Prevalence of Mental Disorders in Children Living in Alberta, Canada, as Determined From Physician Billing Data. Archives of Pediatrics \& Adolescent Medicine. 2001;155(10):1153-9. DOI: 10.1001/archpedi.155.10.1153.

27. Yu P. Mortality of children and parental disadvantage. Australian Social Policy No 7. 2008:1.

28. Yu P. Sequence matters: understanding the relationship between parental income support receipt and child mortality. Australian Social Policy Journal. 2010(9).

29. Comeau J, Duncan L, Georgiades K, Wang L, Boyle MH. Social assistance and trajectories of child mental health problems in Canada: evidence from the National Longitudinal Survey of Children and Youth. Can J Public Health. 2020 Aug;111(4):585-93. DOI: 10.17269/s41997-020-00299-1.

30. Lee H, Harris KM, Lee J. Multiple levels of social disadvantage and links to obesity in adolescence and young adulthood. The Journal of school health. 2013;83(3):13949. DOI: $10.1111 /$ josh.12009.

31. Hernán MA, Hsu J, Healy B. A Second Chance to Get Causal Inference Right: A Classification of Data Science Tasks. CHANCE. 2019 2019/01/02;32(1):42-9. DOI: 10.1080/09332480.2019.1579578.

32. Walter M, Lovett R, Maher B, Williamson B, Prehn J, Bodkin-Andrews G, et al. Indigenous Data Sovereignty in the Era of Big Data and Open Data. Australian Journal of Social Issues. 2021;56(2):143-56. DOI: https://doi.org/10.1002/ajs4.141.

33. Pilkington R, Montgomerie M, Grant J, Gialamas A, Malvaso C, Smithers L, et al. An innovative linked data platform to improve the wellbeing of children - the South 
It is made available under a CC-BY-NC-ND 4.0 International license .

Australian Early Childhood Data Project. Australia's welfare series no. 14. Cat. no. AUS 226. Canberra. ; 2019.

34. SA-NT DataLink: Supporting health, social and economic research, education and policy in South Australia and the Northern Territory. Adelaide: University of South Australia; 2018 [cited 2016 20th March]. Available from:

https://www.santdatalink.org.au/

35. Holman CD, Bass AJ, Rouse IL, Hobbs MS. Population-based linkage of health records in Western Australia: development of a health services research linked database. Aust N Z J Public Health. 1999 Oct;23(5):453-9.

36. Centre for Health Record Linkage. Quality assurance. Sydney, Australia: NSW Ministry of Health; 2012. Available from: http://www.cherel.org.au/quality-assurance

37. Australian Insitute of Health and Welfare. Data available from the Department of Social Services. AIHW; 2018 [cited 2019 09/06/2019]. Available from: https://www.aihw.gov.au/our-services/data-on-request/dss-data

38. Gialamas A, Pilkington R, Berry J, Scalzi D, Gibson O, Brown A, et al. Identification of Aboriginal children using linked administrative data: Consequences for measuring inequalities. Journal of Paediatrics and Child Health. 2016;52(5):534-40.

39. Malvaso CG, Delfabbro PH, Day A, Nobes G. Young People Under Youth Justice Supervision With Varying Child Protection Histories: An Analysis of Group Differences. International Journal of Offender Therapy and Comparative Criminology. 2019;63(2):159-78. DOI: 10.1177/0306624x18791735.

40. Procter AM, Pilkington RM, Lynch JW, Smithers LG, Chittleborough CR. Potentially preventable hospitalisations in children: a comparison of definitions. Archives of Disease in Childhood. 2020;105(4):375-81. DOI: 10.1136/archdischild-2019-316945.

41. Procter AM, Gialamas A, Pilkington RM, Montgomerie A, Chittleborough CR, Smithers LG, et al. Characteristics of paediatric frequent presenters at emergency departments: A whole-of-population study. Journal of Paediatrics and Child Health. 2021;57(1):64-72. DOI: 10.1111/jpc.15119.

42. Hernán MA, Robins JM. Using Big Data to Emulate a Target Trial When a Randomized Trial Is Not Available. American Journal of Epidemiology. 2016;183(8):758-64. DOI: 10.1093/aje/kwv254.

43. Select Committee on Intergenerational Welfare Dependence. Living on the Edge: Inquiry into Intergenerational Welfare Dependence. Canberra: Commonwealth of Australia; 2019.

44. Weier M, Dolan K, Powell A, Muir K, Young A. Money Stories: Financial resilience among Aboriginal and Torres Strait Islander Australians. 2019.

45. Australian Institute of Health and Welfare. Admitted patient care 2017-18: Australian hospital statistics (Cat. no. HSE 225; Health services series no. 90). Canberra: AIHW, 2019. 